\title{
The relationship between the alkaline phosphatase network and the haematopoiesis in mice subjected to whole-body irradiation
}

Khaled M. Almohamad, Fatima M. Alsheikh

\begin{abstract}
Purpose: To investigate the relationship between the alkaline phosphatase (ALP) network of the marrow stroma and the haematopoietic regeneration after mice whole-body irradiation. Materials and methods: Three groups of mice were irradiated with a non-lethal ionising radiation dose: the first one received an intraperitoneal injection of Levamisole, ALP inhibitor, $24 \mathrm{~h}$ before irradiation; the second one received an intraperitoneal injection of Lisinopril, haematopoiesis inhibitor, $24 \mathrm{~h}$ before irradiation; the third was left untreated, but irradiated. The fourth group, untreated and not irradiated, was the control. The total surface occupied by ALP positive processes, revealed by means of ALP cytochemistry in the marrow area, was evaluated semi-quantitively. Nucleated bone marrow cells were also counted. Results: ALP network began to increase $24 \mathrm{~h}$ after irradiation to reach a maximum after $72 \mathrm{~h}$, when the bone marrow was almost become completely empty of the haematopoietic cells. This increase advances the haematopoietic recovery. This process was substantially delayed when the mice were injected with Levamisole $24 \mathrm{~h}$ before irradiation. On the contrary, ALP network increased strongly since the first day after irradiation when the mice were injected with Lisinopril $24 \mathrm{~h}$ before irradiation. Conclusions: These data have indicated that the haematopoietic recovery and repopulation of the bone marrow were advanced by the ALP network recovery.
\end{abstract}

Key words: haematopoiesis $\bullet$ whole-body irradiation $\bullet$ alkaline phosphatase (ALP) network $\bullet$ Levamisole $\bullet$ Lisinopril

K. M. Almohamad ${ }^{\bowtie}$, F. M. Alsheikh

Hematological Diseases Laboratory,

Department of Radiation Medicine,

Atomic Energy Commission of Syria (AECS),

P. O. Box 6091 Damascus, Syria,

Tel.: +96311213 2580, Fax: +963116112289,

E-mail: ascientific@aec.org.sy

Received: 20 February 2014

Accepted: 11 June 2014

\section{Introduction}

The marrow cavity of mammals contains a network of complex supportive stroma consisting of connective tissue and many cell types, including fibroblasts, macrophages, adipocytes, smooth muscle cells, reticular and endothelial cells [1]. These cells are spatially arranged in a three-dimensional architecture in which haematopoietic cells are distributed, clustered or separated, forming an array of islands [2].

Marrow stromal cells seem to communicate with haematopoietic cells through cell-to-cell signals originating from cytokines, membrane-bound molecules, extra-cellular matrix [3, 4] and gap junctions [5]. Such communication between marrow stromal cells and haematopoietic cells is believed to regulate homing, cell survival, proliferation and mobilisation of blood cells. Previous observations indicate that the bone marrow stromal reticular fibroblasts (Westen-Baiton cells) [6] seem to play a role in haematopoiesis. Morphological studies carried out using light, electron and confocal microscopy showed that these cells can develop long prolongations in contact with cells of myeloid lineage and form 
myelopoiesis-environmental 'niches' [7]. Because of this particular morphology, these cells are often referred to as 'reticular' cells and the network they form is known as the alkaline phosphatase (ALP) network. The extent of this network is modified in some conditions. In acute leukaemia, the network of ALP-positive cells is much more dense than that in the normal marrow [8]; on the contrary, ALP expression is low after bone marrow transplantation and is associated with depressed haematopoiesis [9]. Recovery of the ALP network in aplastic bone marrow is partly due to the recruitment of ALP-positive cells. It advances the haematopoietic recovery and consequently, the equilibrium between fat cells and ALP-positive cells seems to be controlled by haematopoietic cells [10].

In this study, we used a model of radiation-induced bone marrow aplasia in mice in order to confirm the relationship between ALP network evolution and haematopoietic recovery.

\section{Materials and methods}

\section{Animals}

Six-week-old OF1 mice were used. The animals were handled according to the recommendations of the declaration of Helsinki and the internationally accepted principles for the care and use of experimental animals. The OF1 strain was initially obtained from Charles River Laboratories, France; then, it was bred and raised in the animal house of our facilities. The mice were kept in a clean conventional environment and given nutritional chow (typical rodent diets, containing crude protein $18-20 \%$, crude fat $5-7 \%$, carbohydrates $60-65 \%$ and $3700 \mathrm{kcal} / \mathrm{kg}$ of metabolisable energy) and water ad libitum. The mice were maintained on a 7 a.m. to 7 p.m. light-dark cycle.

\section{Irradiation and injection procedures}

Four Gy acute whole-body irradiation $(116 \mathrm{mGy} / \mathrm{m})$ was administered to mice using gamma ray apparatus (Theratron 80 Canadian design machine, Co-60, focal distance of $100 \mathrm{~cm}$ ) located in our laboratories. Mice whole-body irradiation was carried out in special irradiation-cages, containing from 10 to 15 mice at the same time. The first group has received one intraperitoneal injection of Lisinopril $(10 \mathrm{mg} / \mathrm{kg}$ mouse body weight), an inhibitor of proliferation of the haematopoietic stem cells [11]. The second group has received one intraperitoneal injection of Levamisole $(10 \mathrm{mg} / \mathrm{kg}$ mouse body weight), an ALP inhibitor [12, 13]. The injections were given $24 \mathrm{~h}$ before irradiation.

\section{Tissue processing for histology}

The irradiated mice were killed under anaesthesia by cervical dislocation at different times $(1,3$, 7, 14 and 30 days) after irradiation. As controls, non-irradiated mice were killed at 1, 3, 7, 14 and
30 day-endpoints. Three mice have been used for each endpoint. From each animal, both femurs were removed, cut longitudinally and fixed in Histocon ${ }^{\circledR}$ (Polysciences Inc., USA), embedded in cryostat embedding medium (Killik, Bio-Optica, Italy) and frozen at $-80^{\circ} \mathrm{C}$ or fixed in $10 \%$ neutral buffered formalin (Panreac, Spain), cleaned in Xylene (VWR International, UK) and embedded in paraffin. Spleen preparations were prepared by the same procedures. Five micrometre cryostat sections and $4 \mu \mathrm{m}$ paraffin sections were made in order to cover the whole marrow cavity from one side of the cortical bone to the other side.

\section{Counting of bone marrow cells}

Three mice were used for each endpoint and two femurs of each mouse were removed. The femur was removed, both extremities were cut. The bone marrow was flushed with the medium culture (DMEM, Biochrom, Germany) using a syringe and a 21-Gauge needle; the suspension was filtered through a piece of nylon (Blutex no. 37, $70 \mu \mathrm{m}$, Saatitech, Italy) and centrifuged. The cells were resuspended in the medium and counted using a Thoma hemocytometer.

\section{ALP activity detection}

For ALP activity detection, the $5 \mu \mathrm{m}$ cryostat sections were incubated $20 \mathrm{~min}$ at $37^{\circ} \mathrm{C}$ in a reaction medium containing $1 \mathrm{mg} / \mathrm{mL}$ of Fast Blue BB Salt (Sigma, UK), $0.3 \mathrm{mg} / \mathrm{mL}$ of Naphtol-AS-Phosphate (Sigma, UK) and $0.5 \%$ of $N, N$-dimethylformamide in $0.2 \mathrm{M}$ Tris buffer ( $\mathrm{pH}$ 9.1). Negative controls were incubated with the Naphtol-AS-Phosphate solution without the Fast Blue BB Salt.

\section{Images capturing and analysis}

Images were taken using a light microscope (BX51, Olympus, Japan) linked to a personal computer. Images for cell evaluation and ALP semi-quantification were taken with $40 \times$ objectives. The ALP network surface of the marrow area in each field was semi-quantified, following a slight modification of an already described procedure [14]. For each endpoint, three mice were used and a minimum of 10 different fields per femur were analysed.

\section{Results}

\section{ALP network in the normal bone marrow}

In normal bone marrow mouse, the marrow cellularity covers the whole bone marrow cavity and the number of the nucleated bone marrow is about 53 million cells for both mouse femurs (Table 1). ALP activity is mainly observed in the paratrabecular areas, in close contact with cells of the myeloid lineage. The surface of ALP network was evaluated semi-quantitively in 


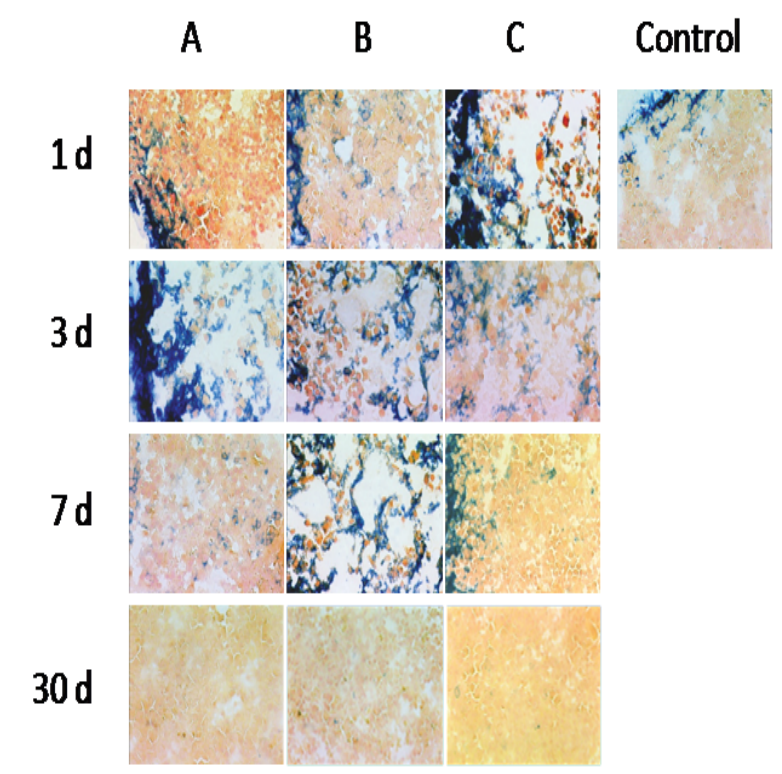

Fig. 1. ALP activity (revealed in blue by Fast Blue BB Salt) estimated on embedded bone marrow cryostat sections. ALP activity in bone marrow area (A) reached a maximum 3 days after irradiation; then, it started to decrease. In mice that were Levamisole-injected $24 \mathrm{~h}$ before irradiation (B), ALP activity reached a maximum with some delay, on days 3-7 after irradiation; then, it started to decrease. In mice that were Lisinopril-injected $24 \mathrm{~h}$ before irradiation (C), ALP activity reached a maximum so early on day 1 after irradiation; then, it started to decrease. (Original magnification $\times 400$ ).

the marrow area. At least 10 different images per femur were analysed for three mice at each endpoint and the ALP network surface was observed, as shown in Fig. 1 and Table 2.
Bone marrow cellularity, ALP network evolution after irradiation

After a 4 Gy whole-body irradiation, the bone marrow cellularity, as assessed by morphology and confirmed by counting the nucleated bone marrow cells, decreased during the first 5 days after irradiation. Then, it started to increase and the aspect of bone marrow appeared similar to that of the controls between days 14 and 30 (Table 1 ). Therefore, the results of day 14 did not appear in the tables and figures, because they were so close to results of day 30 . On day 1 after irradiation, the ALP network surface was similar to that observed in the non-irradiated control mice. This surface was then significantly increased to reach maximum values on day 3 after irradiation (approximately +++ ) and returned to normal values on day 30 (Table 2 and Fig. 1). Interestingly, this network that is essentially concentrated in the paratrabecular area in normal conditions started to spread throughout the bone marrow.

\section{Bone marrow cellularity and ALP network evolution} after irradiation in the Levamisole-injected mice

The bone marrow cellularity in mice Levamisole-injected $24 \mathrm{~h}$ before irradiation, as assessed by morphology and confirmed by counting the nucleated bone marrow cells, was strongly decreased on day 3 after irradiation. Then, it started to increase with some delay, as compared to the case of non-injected irradiated mice (Table 1). One day after the irradiation, the ALP network surface was poorly visible. Its surface was then significantly increased and reached maximum values on day 7 after irradiation (approximately

Table 1. Number of nucleated bone marrow cells for both mouse femurs

\begin{tabular}{|c|c|c|c|c|}
\hline Time/days ${ }^{\#}$ & Non-irradiated & Irradiated* & Irradiated-Leva $^{\dagger}$ & Irradiated-Lisino $^{\neq}$ \\
\hline 1 & $53.33 \pm 4.16$ & $61.00 \pm 5.00$ & $56.00 \pm 3.40$ & $34.00 \pm 3.10$ \\
\hline 3 & $53.67 \pm 2.52$ & $17.50 \pm 0.10$ & $14.00 \pm 0.60$ & $66.00 \pm 6.30$ \\
\hline 7 & $52.67 \pm 3.79$ & $59.00 \pm 4.50$ & $47.00 \pm 1.80$ & $40.00 \pm 4.64$ \\
\hline 30 & $53.00 \pm 2.00$ & $45.00 \pm 1.00$ & $53.00 \pm 2.70$ & $64.00 \pm 5.50$ \\
\hline
\end{tabular}

Each value represents the data for at least three mice. Results are expressed in millions. Standard deviation indicated.

\# Control mice were sacrificed in parallel to the irradiated mice. The data obtained for the day after irradiation as indicated.

"Values represent the number of nucleated bone marrow cells in irradiated mice.

+ Values represent the number of nucleated bone marrow cells in mice that were Levamisole-injected $24 \mathrm{~h}$ before irradiation.

${ }^{\neq}$Values represent the number of nucleated bone marrow cells in mice that were Lisinopril-injected $24 \mathrm{~h}$ before irradiation.

Table 2. Semi-quantification of ALP network surface in marrow area

\begin{tabular}{lcccc}
\hline & \multicolumn{5}{c}{ ALP network } \\
\cline { 2 - 5 } Time/days $^{\#}$ & control & irradiated & irradiated-Leva $^{\dagger}$ & irradiated-Lisino $^{*}$ \\
\hline 1 & + & + & + & +++ \\
3 & + & +++ & ++ & ++ \\
7 & + & ++ & +++ & ++ \\
30 & + & + & + & + \\
\hline
\end{tabular}

Each value represents the data for three mice (between 10 and 20 microscopic fields per mouse were reviewed). Normal ALP network $(+)$, medium ALP network $(++)$ and high ALP network $(+++)$.

${ }^{\#}$ Control mice were sacrificed in parallel to the irradiated mice. The data obtained for the day after irradiation as indicated.

"Values represent the ALP network surface per field in irradiated mice.

†Values represent the ALP network surface per field in mice that were Levamisole-injected $24 \mathrm{~h}$ before irradiation.

${ }^{*}$ Values represent the ALP network surface per field in mice that were Lisinopril-injected 24 h before irradiation. 
+++ ), subsequently returning to normal values on day 30 (Table 2 and Fig. 1).

Bone marrow cellularity and ALP network evolution after irradiation in the Lisinopril-injected mice

The bone marrow cellularity in mice that were Lisinopril-injected $24 \mathrm{~h}$ before irradiation, as assessed by morphology and confirmed by counting the nucleated bone marrow cells, was decreased slightly 1 day after irradiation. Then, it started to increase, reaching a maximum value on day 3 after irradiation (Table 1). On day 1 after irradiation, the ALP network surface was maximally observed (approximately +++ ). The surface then decreased to reach values near to normal on day 7 after irradiation (Table 2 and Fig. 1).

\section{ALP network evolution in spleen}

The ALP network in spleen showed exactly the same behaviour as that in the bone marrow area and it followed the same evolution after irradiation alone and combined with Levamisole or Lisinopril injection (Fig. 2).

\section{Discussion}

Many studies have described the bone marrow haematopoietic regeneration post-chemotherapy

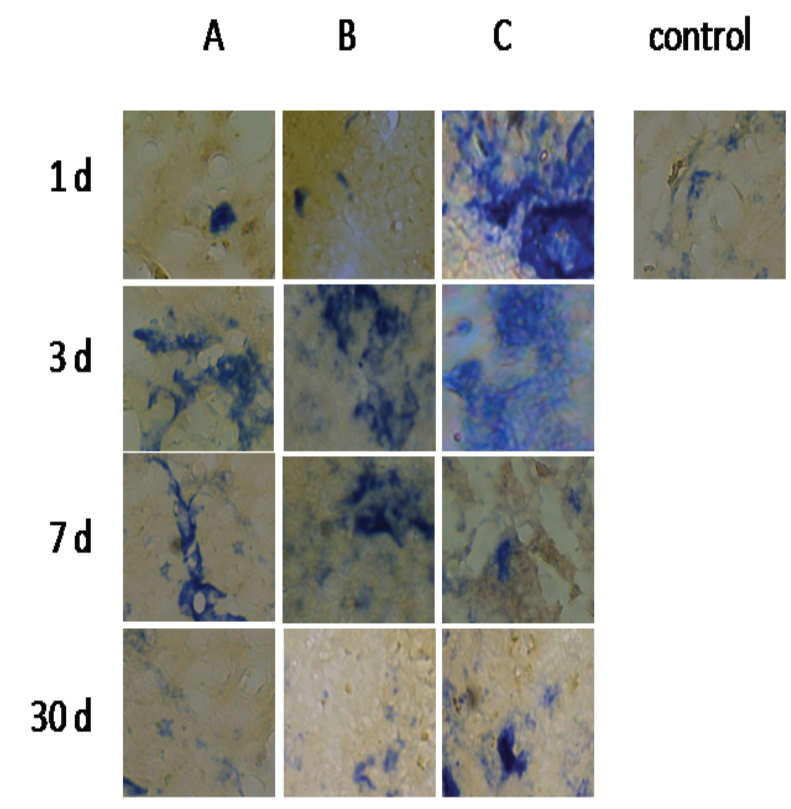

Fig. 2. ALP activity (revealed in blue by Fast Blue BB Salt) estimated on embedded spleen cryostat sections. ALP activity in the spleen (A) reached a maximum 3 days after irradiation; then, it started to decrease. In mice that were Levamisole-injected $24 \mathrm{~h}$ before irradiation (B), ALP activity reached a maximum with some delay, on days 3-7 after irradiation; then, it started to decrease. In mice that were Lisinopril-injected $24 \mathrm{~h}$ before irradiation (C), ALP activity reached a maximum early on day 1 after irradiation; then, it started to decrease. (Original magnification $\times 400)$. and post-bone marrow transplantation [15]. Stromal regeneration and, particularly, the evolution of haematopoietic niches have not yet been fully and extensively described. Such an analysis could be helpful to understand the function of these cells in supporting the haematopoiesis as well as their role under pathological conditions.

In our previous study, we analysed the recovery of the bone marrow stroma, especially the ALP-positive cells, within a model of radio-induced medullary aplasia. An image analysing system was used to quantify the surface of ALP positive processes (ALP network) and to count the number of ALP-positive cells using the nuclei as the defining unit on Fast Blue BB Salt stained sections from both irradiated and non-irradiated control mice [10]. This model permitted to quantify the surface of the ALP network and to follow its evolution after irradiation. This work has been designed to investigate the relationship between the ALP network of the marrow stroma and spleen and the haematopoietic regeneration in mice after whole-body irradiation.

Two inhibitors were used. The first one, Levamisole, is an ALP inhibitor [12, 13] which reduces the ALP expression that precedes the bone marrow recovery occurring after whole-body irradiation [10]. Thus, the effect of ALP reduction on the bone marrow recovery can be monitored. The second one, Lisinopril, a haematopoiesis inhibitor [11], is used to suppress the bone marrow recovery after whole-body irradiation. Hence, the effect of the bone marrow cellularity suppression on the ALP expression can be monitored.

The experimental data indicated that the ALP-positive cells and the ALP network were scarce in the normal marrow and irregularly distributed across marrow spaces $[2,9,10]$. In the non-injected mice, in the first few days after irradiation the density of the ALP network increased in the marrow area. The increase preceded the haematopoietic bone marrow regeneration which started on day 5 after irradiation, as assessed morphologically. It was confirmed by counting the nucleated bone marrow cells in this work $(59 \pm 4.5$ million cells on day 7$)$ and these results are in a good agreement with the previous studies $[10,16,17]$. Others have reported an increase in the number of reticular-adventitial cells within 2 days of hypertransfusion [18]. Those authors used repeated transfusion in normal mice to suppress the erythropoiesis, and they have related this observation to a shift in the marrow's microenvironment simultaneous to a shift from erythropoiesis to granulopoiesis.

On day 1 after irradiation, in mice that were Levamisole-injected $24 \mathrm{~h}$ before irradiation, the ALP network surface was scarce. The surface then significantly increased to reach the maximum value on day 7 after irradiation (approximately +++ ); it returned to a normal value on day 30 (Table 2 and Fig. 1). The bone marrow cellularity, as assessed by morphology and confirmed by counting of the nucleated bone marrow cells, was strongly decreased on day 3 after irradiation ( $14 \pm 0.6$ million cells); then, it started to increase with some delay, as compared 
to the case of non-injected irradiated mice following the development of the ALP network (Table 1).

The bone marrow cellularity in mice that were Lisinopril-injected $24 \mathrm{~h}$ before irradiation, as assessed by morphology and confirmed by bone marrow cell counting, decreased slightly on day 1 after irradiation. Then, it started to increase, reaching a maximum on day 3 after irradiation (Table 1) following the ALP network development that was clearly seen (approximately +++$) 24 \mathrm{~h}$ post-irradiation. Its surface then decreased to reach values near to normal on day 7 post-irradiation (Table 2 and Fig. 1). These observations indicate that the erythropoiesis inhibition by Lisinopril possibly stimulates the ALP-positive cells. Thus, the ALP network surface considerably increases and this ALP increase is followed by augmentation of the nucleated bone marrow cells. These observations are in agreement with the previous studies which indicated that the abnormal haematopoietic cells seemed to be unable to control this network: the ALP-positive cells were very abundant in the untreated acute non-lymphoid leukaemia [14] or acute myeloid leukaemia [19].

It is well known that spleen in the rodents participates in haematopoiesis, especially in the granulopoiesis. Hence, the ALP network in spleen displayed same pattern of evolution as that in the marrow (Fig. 2).

In this work, we report for the first time that alteration of the ALP activity in the irradiated mice leads to clear-cut changes in the bone marrow cellularity that are always preceded by the ALP network increase. Nevertheless, it is still unknown, whether the increase in the ALP network is due to a stimulation of the ALP-positive cells, a recruitment of the ALP-positive cells, or to an activation of the expression of the ALP genes in the marrow cells. This is a very important issue. Firstly, we have to be certain that a sufficient number of ALP-positive cells, or more precisely that a sufficient number of cells, which can be stimulated to express ALP, is used for bone marrow transplantation. Secondly, if the bone marrow stromal cells, especially ALP-positive cells and/or their precursors, were seriously damaged in certain types of aplasia, then, the bone marrow may not be able to recover. In a recent study, a hypothesis has been put forward that the osteoblast lineage (ALP-positive cells) after irradiation engages in a reversible way and returns to a less differentiated stage [20]. We think that further studies, for example, the analysis of the ALP gene expression after irradiation, are needed to fully understand this issue.

\section{Conclusion}

The results of this work indicate that the increase in the ALP network precede the haematopoietic recovery. By controlling this network by Levamisole (ALP inhibitor) or Lisinopril (erythropoiesis inhibitor) treatment, we demonstrated a clear-cut relationship between the ALP network kinetics and the repopulation of the bone marrow.
Acknowledgment. We thank Hasan Omran and Hadi Al-Tarboush for their technical assistance.

\section{References}

1. Mayani, H., Guilbert, L., \& Janowska-Wieczorek, A. (1992). Biology of the hemopoietic microenvironment. Eur. J. Haematol., 49, 225-233.

2. Bianco, P., \& Boyde, A. (1993). Confocal images of marrow stromal (Westen-Bainton) cells. Histochemistry, 100, 93-99.

3. Kittler, E. L., McGrath, H., Temeles, D., Crittenden, R. B., Kister, V. K., \& Quesenberry, P. J. (1992). Biologic significance of constitutive and subliminal growth factor production by bone marrow stroma. Blood, 79, 3168-3178.

4. Long, H. W. (1992). Blood cell cytoadhesion molecules. Exp. Hematol., 20, 288-301.

5. Krenacs, T., \& Rosendaal, M. (1998). Connexin 43 gap junctions in normal, regenerating and cultured mouse bone marrow and in human leukemia: their possible involvement in blood formation. Am. J. Pathol., 152, 993-1004.

6. Westen, H., \& Baiton, D. F. (1979). Association of alkaline-phosphatase-reticulum cells in bone marrow with granulocytic precursors. J. Exp. Med., 150, 919-937.

7. McKenna, R. W., \& Allison, P. (1990). Diagnosis, classification, and course of myelodysplasic syndromes. Clin. Lab. Med., 10, 683-706.

8. Bianco, P., Bradbeer, J. N., Riminucci, M., \& Boyde, A. (1993). Marrow stromal (Western-Bainton) cells: identification, morphometry, confocal imaging and changes in disease. Bone, 14, 315-320.

9. Dilly, S. A., \& Jagger, C. J. (1990). Bone marrow stromal cell changes in haematological malignancies. J. Clin. Pathol., 43, 942-947.

10. Almohamad, K., Thiry, A., Hubin, F., Belaid, Z., Humblet, C., Boniver, J., \& Defresne, M. -P. (2003) Marrow stromal cell recovery after radiation-induced aplasia in mice. Int. J. Radiat. Biol., 79(4), 259-267.

11. Rousseau-Plasse, A., Wdzieczak-Bakala, J., Lenfant, M., Ezan, E., Robinson, S., Briscoe, C. V., Melville, J., \& Riches, A. (1998). Lisinopril, an angiotensin I-converting enzyme inhibitor, prevents entry of murine hematopoietic stem cells into the cell cycle after irradiation in vivo. Exp. Hematol., 26, 1074-1079.

12. Fallon, M. D., Whyte, M. P., \& Teitelbaum, S. L. (1980). Stereospecific inhibition of alkaline phosphatase by L-tetramisole prevents in vitro cartilage calcification. Lab. Invest., 43, 489-494.

13. Scher, B. M., Fuksina, I., Hellinger, N., Waxman, S., \& Scher, W. (1998). The phosphatase inhibitors, orthovanadate and levamisole, inhibit induction of erythroid differentiation and abrogate the associated inhibition of glycolysis. Int. J. Oncol., 12, 987-996.

14. Bianco, P., Costantini, M., Dearden, L. C., \& Bonucci, E. (1988). Alkaline phosphatase positive precursors of adipocytes in the human bone marrow. Br. J. Haematol., 68, 401-403.

15. Prince, M. H., Simmons, P. J., Whitty, G., Wall, D. P., Barber, L., Toner, G. C., Seymoour, R. F., Richardson, G., Mrongovius, R., \& Haylock, D. N. (2004). Improved hematopoietic recovery following transplantation with ex vivo-expanded mobilized blood cells. $\mathrm{Br}$. J. Haematol., 126(4), 536-545.

16. Haot, J., \& Barakina, N. F. (1969). Quantitative study of the hemopoietic recovery after a sublethal X-irradiation in the mouse. Acta Haematol., 42, 347-356. 
17. Imai, Y., \& Nakao, I. (1987). In vivo radiosensitivity and recovery pattern of the hematopoietic precursor cells and stem cells in mouse bone marrow. Exp. Hematol., 15, 890-895.

18. Brookoff, D., \& Weiss, L. (1982). Adipocyte development and the loss of erythropoietic capacity in the bone marrow of mice after sustained hypertransfusion. Blood, 60, 1337-1344.
19. Dilly, S. A., Jagger, C. J., \& Sloane, J. P. (1993). Stromal cell populations in necropsy bone marrow sections from allogeneic marrow recipients and non-transplant patients. J. Clin. Pathol., 46, 611-616.

20. Poncin, G., Beaulieu, A., Humblet, C., Thiry, A., Oda, K., Boniver, J., \& Defresne, M. -P. (2012). Characterization of spontaneous bone marrow recovery after sublethal total body irradiation: importance of the osteoblastic/adipocytic balance. PLoS One 7:e30818. 